\title{
Effects of Lattice Spacing on Strain Analysis using Phase Technique
}

\author{
W. J. Chang and T. D. Brown
}

Department of Electrical and Computer Engineering, Michigan State University, East Lansing, MI 48824

High-Resolution Transmission Electron Microscopy (HRTEM) is an important tool for the investigation of local deformations in crystal structures. The determination of strain in epitaxially grown layers from HRTEM micrographs can be performed when digital images are obtained using a CCD in the image plane. For strain analysis, the phase technique has been used by several groups [15] to provide 2-D mapping of strain information. The phase technique can be divided into two methods: Geometric Phase Analysis (GPA), proposed by Hytch [1] and Computational Fourier Transform Moiré (CFTM) [2]. The primary difference between these techniques is that the GPA method utilizes the phase image to obtain strain information, while the CFTM method directly calculates strain information by the inverse Fourier transform of a digital diffractogram.

The reliability of the phase technique for small lattice-mismatched systems with a few monolayers has not been previously investigated in detail, even though characterization of small latticemismatched systems is essential to the development of novel device structures. E. Guerrero et al. [6] investigated the error in strain measurements using GPA due to overshoot and undershoot near heterointerfaces in thick In $\mathrm{As}_{\mathrm{x}} \mathrm{P}_{1-\mathrm{x}}$ epilayers on InP, but attributed these features to sample thickness and TEM defocus. In this correspondence, we demonstrate that undershoot at strained heterointerfaces is also dependent on resolution (i.e. number of pixels per lattice spacing) of images. Characterization of undershoot has been performed on strain measurements in terms of the resolution of images and the size of the mask used for Fourier filtering.

In this work, we perform strain analysis on two cases of lattice contraction with four monolayers each, resulting in a strain of $-5 \%$ and $-10 \%$. Strained layers were positioned in the middle of the HRTEM images (See Fig. 1). For the case of a heterointerface of $\mathrm{GaAs}_{1-\mathrm{x}} \mathrm{P}_{\mathrm{x}}$ on a GaAs substrate, we can expect lattice contraction on the $\mathrm{GaAs}_{1-\mathrm{x}} \mathrm{P}_{\mathrm{x}}$ layers to be from zero to $-6 \%$ as the phosphorus composition $(x)$ is varied from zero to $100 \%$. Fig. 1 shows a 2-D lattice image generated using a sinusoidal function along both $\mathrm{x}$ and $\mathrm{y}$ directions, resulting in crossing lattice fringes displaying dot contrast. The CFTM method was used for strain analysis. The results are shown in Fig. 2(a), where a rippled profile is observed within both unstrained regions and at heterointerfaces between strained and unstrained regions. The rippled profiles at the heterointerfaces are exhibited in the form of overshoot and undershoot, a phenomenon resulting from the leakage effect during Fourier transform.

Undershoot is a key factor influencing determination of the maximum strain at heterointerfaces. It is evident from Fig. 2(b) that the maximum measured strain has a linear relationship with mask size for region- 2 in the case of 7 pixels and 12 pixels per lattice fringe, resulting in a large strain error. However, the maximum measured strain is close to the theoretical value of $(-5 \%)$ for region-1 in the case of 20 pixels per lattice spacing, demonstrating improved accuracy. This indicates that undershoot in the strain profile and the associated strain error is minimized with improved resolution of the HRTEM image. In addition, Fig. 2(b) shows that for region-1 the maximum strain approaches the theoretical value $(-5 \%)$ and saturates. This depends directly on the resolution of the HRTEM image. Figure 2(c) shows the maximum strain versus mask size when lattice contraction is $10 \%$, 
resulting in stable maximum strain. Thus, for large lattice contraction, reliable strain measurement is readily achieved.

In conclusion, lattice spacing influences the reliability of the phase technique. The range over which the phase technique can be reliably applied becomes narrow as lattice spacing decreases. Selection of a small mask may be used to remove undershoot at the cost of spectral resolution. For example, if we select a mask size around region-1, we can remove undershoot, resulting in an experimental value of maximum strain which is roughly close to the theoretical value. Results obtained for real HRTEM images will be discussed.

\section{References}

[1] M.J. Hytch and M. Gandais, Philos. Mag. A 72 (1996) 619.

[2] H.C. Choi et al., IBM J. Res. Dev. 37 (1993) 649.

[3] M.J. Hytch et al., Nature 423 (2003) 270.

[4] S. Kret et al., J. Phys.-Condens. Mat. 12 (2000) 10313.

[5] G. Ade, Microelectron. Eng. 51-52 (2000) 3.

[6] E. Guerrero et al., Microsc. Microanal. 13 (2007) 320.

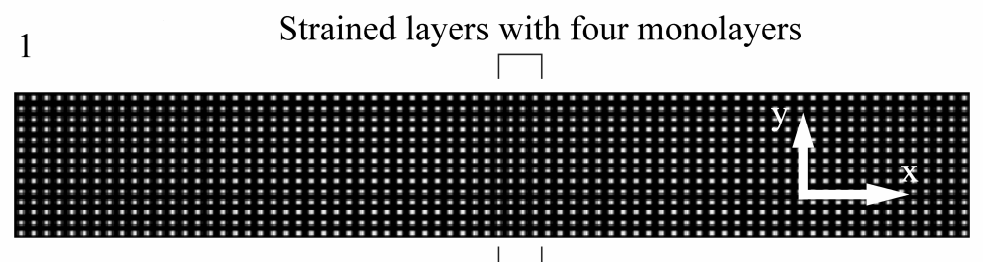

Figure 1. A computer-generated image with 2-D lattice using Matlab. Strained layers with four monolayers were placed in the middle of the image. Arrows indicate coordinate system of $x$ and $y$ axes. Image size is 100 by 600 pixels.

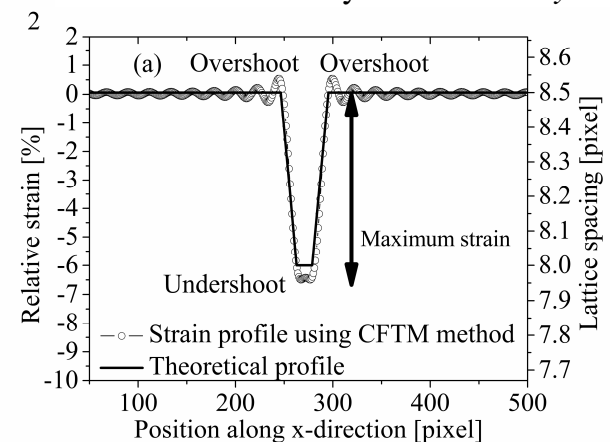

Figure 2. Simulation results using CFTM method with computer-generated images. (a) Strain profile $\left(\varepsilon_{\mathrm{xx}}\right)$ obtained from analysis of HRTEM image. Theoretical profile plotted using a solid line. (b) Maximum strain at heterointerface according to mask size and fringe spacing for a lattice contraction of $-5 \%$. (c) Maximum strain and standard deviation at heterointerface according to mask size for a lattice contraction of $-10 \%$.
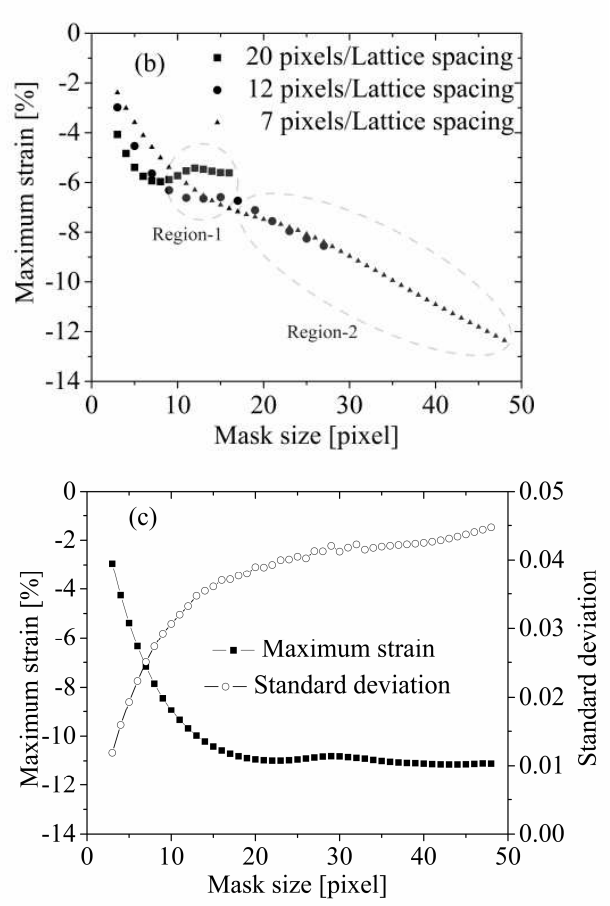